\title{
Fully automatic and segmentation-robust classification of breast tumors based on local texture analysis
}

\author{
Bo Liu $^{1}$ H. D. Cheng ${ }^{1,2}$ Jianhua Huang ${ }^{1}$ Jiafeng Liu ${ }^{1}$ \\ ${ }^{1}$ School of Computer, Harbin Institute of Technology, Harbin, China \\ ${ }^{2}$ Department of Computer Science, Utah State University, Logan, UT 84322 U.S.A
}

\begin{abstract}
In this paper, a novel fully automatic classification method of breast tumors using ultrasound (US) image is proposed. The proposed method can be divided into two steps: "ROI generation step" and "ROI classification step". In the ROI generation step, the proposed method focuses on finding a credible ROI instead of finding the precise location of the breast tumor. In the ROI classification step, local textures in the ROI are considered with a novel strategy. Both steps were implemented by utilizing supervised texture classification approach. The experiments demonstrate that the proposed method is effective and useful for classifying breast tumors.
\end{abstract}

Keywords: texture classification, support vector machine (SVM), computer aided diagnosis, breast ultrasound (BUS) imaging

\section{Introduction}

Due to its noninvasive, practically harmless, and cost effective characteristics, breast ultrasound (BUS) imaging has become one of the most effective and popular approaches for the early detection of breast cancer [1]. However, the interpretation of sonography depends on radiologists' skill and experience. Computer aided diagnosis (CAD) technology has been investigated. Many studies on this topic have been published [1-5].

In existing approaches, a region of interest (ROI) in the image was usually determined; and the features were extracted from the ROI; then the classifiers were employed for determining the tumors' classes. The most critical step for fully automatic classification of BUS images is locating the ROI: (1) due to the poor quality of the image, segmentation of BUS images is a difficult problem [6]; (2) if the ROIs are different from the real tumor regions, the features of the ROIs may differ dramatically from the features of the corresponding real tumor regions, and may affect the classification.

To solve above problem, a novel approach based on texture classification is proposed in this paper. The proposed approach can be divided into two steps: "ROI generation" and "ROI classification”.

\section{ROI generation method}

The ROI generation method can be divided into three steps: (1) the image will be divided into lattices and the lattices will be classified into two classes: "tumor" and "normal tissue", and the image will become binary; (2) post-processing operations will be performed; (3) a serious of background knowledge based rules will be utilized for determining the ROI.

\subsection{Texture extraction}


The original BUS image will be fuzzified based on maximum fuzzy entropy principle [7]. The fuzzified images are divided into non-overlap lattices; the size of the lattices is $16 \times 16$ pixels determined by experiments.

Co-occurrence matrix is utilized for describing the local texture in each lattice. Each element of the co-occurrence matrix $C_{d, \theta}(i, j, d, \theta)$ is defined as the joint probability of the gray levels $i$ and $j$ separated by distance $d$ and along direction $\theta$ of the image [8]:

$$
C_{d, \theta}(i, j, d, \theta)=\frac{\left\|\left\{\begin{array}{l}
\left(\left(x_{1}, y_{1}\right),\left(x_{2}, y_{2}\right)\right) \\
x_{2}-x_{1}=d \cos \theta, \\
y_{2}-y_{1}=d \sin \theta, \\
I\left(x_{1}, y_{1}\right)=i, I\left(x_{2}, y_{2}\right)=j
\end{array}\right]\right\|}{16 \times 16}
$$

where $\left(x_{1}, y_{1}\right)$ and $\left(x_{2}, y_{2}\right)$ are the pixels in the lattice, $I(\bullet)$ is the gray level of the pixel and $\|\cdot\|$ is the number of the pixel pairs satisfying the intensity and spatial conditions. For each lattice, 16 cooccurrence matrices with four directions $\left(\theta=0^{\circ}, 45^{\circ}, 90^{\circ}, 135^{\circ}\right)$ and four distances $(\mathrm{d}=1,2,3,4)$ are established. For reducing the computation complexity and preserving the details, the number of gray levels for establishing the co-occurrence matrices is 64 .

Four texture descriptors are: entropy (ENT), contrast (CON), sum average (SA) and sum entropy (SENT).

$$
\begin{aligned}
& \operatorname{ENT}(d, \theta)=-\sum_{i=0}^{63} \sum_{j=0}^{63} C_{d, \theta}(i, j, d, \theta)\left(\log C_{d, \theta}(i, j, d, \theta)\right) \\
& \operatorname{CON}(d, \theta)=\sum_{k=0}^{63} k^{2} \times \sum_{i=0}^{63} \sum_{j=0}^{63} \delta(|i-j|, k) C_{d, \theta}(i, j, d, \theta) \\
& S A(d, \theta)=\sum_{i=0}^{126} i C_{\text {joint }}(i) \\
& \operatorname{SENT}(d, \theta)=-\sum_{i=0}^{126} C_{\text {joint }}(i) \log \left(C_{\text {joint }}(i)\right)
\end{aligned}
$$

$$
\begin{aligned}
& \delta(x, y)= \begin{cases}1 & \text { if } x=y \\
0 & \text { if } x \neq y\end{cases} \\
& C_{\text {joint }}=\sum_{i=0}^{63} \sum_{j=0}^{63} \delta(i+j, k) C_{d, \theta}(i, j, d, \theta), k=0 \ldots 126
\end{aligned}
$$

The texture descriptors of the same distance will be averaged to reduce the dimension of the feature vectors, so that there are 16 features extracted from the co-occurrence matrix with two additional features: the mean and the variance of the intensities in a lattice.

\subsection{Classification of the lattices}

For classifying the lattices, kernel support vector machine (KSVM) method is utilized [9].

The class of an unknown sample $\mathbf{x}$ is determined [9]:

$$
F\left(\mathbf{x}, \boldsymbol{\alpha}^{0}\right)=\operatorname{sign}\left(\sum_{l=1}^{S V} y_{l} \alpha_{l}^{0} K\left(\mathbf{x}, \mathbf{x}_{l}\right)+b\right)(6)
$$

where each $x_{l}$ is a support vector obtained from training, and $S V$ is the number of support vectors.

The kernel function is the RBF kernel:

$$
K\left(\mathbf{x}, \mathbf{x}_{l}\right)=\exp \left(-\gamma\left|\mathbf{x}-\mathbf{x}_{l}\right|^{2}\right)
$$

Then a binary image, $I_{c}$, obtained:

$$
I_{c}(x, y)=\left\{\begin{array}{c}
1, \quad \text { if the lattice placed at } \\
\quad(16 \times x, 16 \times y) \text { in } I_{\text {ori }} \\
\text { belongs to "tumor" class } \\
0, \quad \text { if the lattice placed at } \\
(16 \times x, 16 \times y) \text { in } I_{\text {ori }} \\
\text { belongs to "normal tissue" class }
\end{array}\right.
$$

\subsection{Post processing and ROI genera- tion}

Post-processing operations are utilized for eliminating the line-like areas, and filling the holes in $I_{c}$. The eliminating operation will set the pixels in the pixel set defined by Eq. (8) to 0, and the filling op- 
eration will set the pixels in the pixel set defined by Eq. (9) to 1 in $I_{c}$.

$$
\begin{aligned}
& \left\{\begin{array}{l}
(x, y) \mid I_{c}(x, y)=1 \text { AND } \\
\left(\begin{array}{l}
\left(I_{c}(x-1, y)=0 \text { AND } I_{c}(x+1, y)=0\right) \\
\text { OR }\left(I_{c}(x, y-1)=0 \text { AND } I_{c}(x, y+1)=0\right)
\end{array}\right)
\end{array}\right\} \\
& \left\{\begin{array}{l}
(x, y) \mid I_{c}(x, y)=0 \text { AND } \\
\left(\begin{array}{l}
\left.I_{c}(x-1, y)=1 \text { OR } I_{c}(x+1, y)=1\right) \\
\text { AND }\left(I_{c}(x, y-1)=1 \text { OR } I_{c}(x, y+1)=1\right)
\end{array}\right)
\end{array}\right\}
\end{aligned}
$$

An eliminating operation is performed first and filling operation fills holes, then the eliminating operation will be performed again. The resulting image $I_{c o}$ is mapped to the original size which is defined as $I_{c r}$.

As the fat and muscle layers are placed in the upper and lower part of the image, the candidate regions which are too high and too low should be removed. Two thresholds $D_{S F}$ and $D_{M M}$ are employed for removing the candidate regions in the fat and muscle layers. Here, the two thresholds are determined with the radiologist's experience. According to radiologist's experience, $D_{S F}=4 \times 16$ (i.e., four lattice rows) and $D_{M M}=3 / 4 h$, where $h$ is the height of the image, and the regions whose vertical positions of the centroids (i.e., rows of the centroids) are $<D_{S F}$ or $>D_{M M}$ will be removed. Two rules are for generating ROI finally:

(1) Find the region with the largest area from the candidate regions, and remove the regions whose areas smaller than the half of the largest area.

(2) Select the remaining region nearest to the center of the image as the final ROI.

After ROI selection, a new binary image $I_{b r}$ can be obtained from $I_{c r}$, in which only the pixels in the ROI region will be 1 , and other pixels will be set to 0 .

\section{ROI classification method}

When the ROI is determined, a strategy will be employed for classification of the ROI. The classification is done in the following steps:

(1) For each ROI, some points are evenly selected and named the "classification checkpoints".

(2) For each classification checkpoint, five windows are formed. Local texture will be extracted from the five windows, and a feature vector will be composed.

(3) For each classification checkpoint, input its feature vector into a well trained classifier to determine the checkpoint's class (benign or malignant).

(4) The ratio between the number of the malignant checkpoints and the total number of all the checkpoints in a ROI is $P_{C R}$. If $P_{C R}>T_{\text {class }}$, a pre-determined threshold, the tumor will be malignant, otherwise, it will be benign. $T_{\text {class }}$ is selected as 0.5 in the experiments.

\subsection{Placement of the classification checkpoints}

The selection of classification checkpoints in a ROI can be written as a pixel set defined in the US image:

$$
\left\{\begin{array}{c}
(x, y) \mid x \bmod d_{c c}=0, y \bmod d_{c c}=0 \\
,(x, y) \text { in the ROI }
\end{array}\right\}
$$

where $d_{c c}$ is a constant for controlling the number of the checkpoints, and $d_{c c}$ is selected as 8 in the experiments.

\subsection{Feature extraction of the classifi- cation checkpoint}

Feature extraction of classification checkpoint is the core of the proposed classification method, and two factors have to be considered: (1) how to set the feature extraction windows, and (2) what features to extract. 
For a checkpoint at $\left(x_{0}, y_{0}\right)$, five feature extraction windows are set, and defined as the pixel sets below:

$$
\begin{aligned}
& W_{0}\left(x_{0}, y_{0}\right)=\left\{\begin{array}{c}
(x, y)|| x-x_{0} \mid<d_{\text {win }} / 2, \\
\left|y-y_{0}\right|<d_{\text {win }} / 2
\end{array}\right\} \\
& W_{1}\left(x_{0}, y_{0}\right)=\left\{\begin{array}{c}
(x, y)|| x-\left(x_{0}-d_{\text {win }} \mid<d_{\text {win }} / 2,\right. \\
\left|y-y_{\text {win }}\right|<d_{\text {win }} / 2
\end{array}\right\} \\
& W_{2}\left(x_{0}, y_{0}\right)=\left\{\begin{array}{c}
(x, y)|| x-\left(x_{0}+d_{\text {win }}\right) \mid<d_{\text {win }} / 2, \\
\left|y-y_{0}\right|<d_{\text {win }} / 2
\end{array}\right\} \\
& W_{3}\left(x_{0}, y_{0}\right)=\left\{\begin{array}{c}
(x, y)|| x-x_{0} \mid<d_{\text {win }} / 2, \\
\left|y-\left(y_{0}-d_{\text {win }}\right)\right|<d_{\text {win }} / 2
\end{array}\right\} \\
& W_{4}\left(x_{0}, y_{0}\right)=\left\{\begin{array}{c}
(x, y)|| x-x_{0} \mid<d_{\text {win }} / 2, \\
\left|y-\left(y_{0}+d_{\text {win }}\right)\right|<d_{\text {win }} / 2
\end{array}\right\}
\end{aligned}
$$

where $0 \leq x \leq W_{\text {ori }}, 0 \leq y \leq H_{\text {ori }}, W_{\text {ori }}$ and $H_{\text {ori }}$ are the width and height of the BUS image, and $d_{\text {win }}$ is the coefficient for controlling the size of the window.

When the windows are set, cooccurrence matrix will be utilized for modeling the local texture around each checkpoint. The features are extracted as below:

(1) Perform equalization on the BUS images.

(2) Establish co-occurrence matrices with different directions and distances in the five windows, respecitvely.

(3) Calculate the texture descriptors from the established co-occurrence matrices, and the mean and variance of the windows.

(4) Form the feature vector using the results in (3).

There are five distances $(d=1,2,3,4$, 8) for the co-occurrence matrices, defined as $d_{i}, i=1 \ldots 5$. There are four directions $(\theta$ $\left.=0^{\circ}, 45^{\circ}, 90^{\circ}, 135^{\circ}\right)$, as $\theta_{j}, j=1 \ldots 4$. Given the distance $d_{i}$, direction $\theta_{j}$ and window $W_{k}$, the four categories of the descriptors are utilized again: $\quad f_{1}\left(d_{i}, \theta_{j}, W_{k}\right) \quad$ (entropy), $f_{2}\left(d_{i}, \theta_{j}, W_{k}\right)$ (contrast), $f_{3}\left(d_{i}, \theta_{j}, W_{k}\right)$ (sum average), and $f_{4}\left(d_{i}, \theta_{j}, W_{k}\right)$ (sum entropy), where $i=1 . . .5, j=1 . .44, k=0 . . .4$.

The descriptors calculated from different windows of the same checkpoint will be combined. For a given distance $d_{i}$, four kinds of combinations of the descriptors along different directions in the center and neighboring windows are utilized (mean1, mean2, range1, range2):

$$
\begin{aligned}
& f_{s}^{\text {mean1 } 1}\left(d_{i}\right)= \sum_{j=0}^{4} f_{s}\left(d_{i}, \theta_{j}, W_{0}\right) / 4, s=1 \ldots 4 \\
& f_{s}^{\text {mean } 2}\left(d_{i}\right)= \sum_{k=1}^{4} \sum_{j=0}^{4} f_{s}\left(d_{i}, \theta_{j}, W_{k}\right) / 4 \\
&, s=1 \ldots 4, k=1 \ldots 4 \\
& f_{s}^{\text {rangel }}\left(d_{i}\right)=\left|\begin{array}{l}
\max _{\theta}\left\{f_{s}\left(d_{i}, \theta_{j}, W_{0}\right)\right\} \\
-\min _{\theta}\left\{f_{s}\left(d_{i}, \theta_{j}, W_{0}\right)\right\}
\end{array}\right| / 4 \\
&, s=1 \ldots 4, \theta=1 \ldots 4 \\
& f_{s}^{\text {range2 }}\left(d_{i}\right)=\mid \begin{array}{l}
\max _{\theta, W}\left\{f_{s}\left(d_{i}, \theta_{j}, W_{k}\right)\right\} \\
-\min _{\theta, W}\left\{f_{s}\left(d_{i}, \theta_{j}, W_{k}\right)\right\} \mid / 4 \\
, s=1 \ldots . .4, \theta=1 \ldots 4, k=1 \ldots 4
\end{array}
\end{aligned}
$$

Four kinds of combinations of the descriptors are used as the classification features. The mean and the variance will also be utilized, and to each window, they can be defined as $f_{5}\left(W_{k}\right), k=0 \ldots 4$ (mean) and $f_{6}\left(W_{k}\right), k=0 \ldots 4$ (variance). Finally, there are 90 features for each checkpoint.

\subsection{Classification of the tumors}

For classifying the checkpoint, kernel support vector machine (KSVM) method is utilized. As mentioned before, the classifier will determine whether a checkpoint belongs to a malignant or a benign tumor. In a ROI, define the number of the classification checkpoints as $N_{C C P}$, and define the number of the checkpoints classified as "malignant" as $N_{C C P-M}$, then, classification ratio $P_{C R}$ can be calculated: 


$$
P_{C R}=N_{C C P-M} / N_{C C P}
$$

If $P_{C R}>T_{\text {class }}$, the tumor is classified as "malignant", otherwise, it is as "benign".

\section{Experimental Result}

A BUS image database containing 112 BUS images (60 malignant and 52 benign) was used in the experiments. The BUS images were collected by using a VIVID 7 (GE, Horten, Norway) with a 5-14 MHz linear probe, and captured directly from the video signals. The images were obtained from the Second Affiliated Hospital of Harbin Medical University, and all the classes of the images were confirmed by surgery and pathological examinations or biopsy.

Here, k-fold cross validation method is utilized where $\mathrm{k}=11$.

For illustrating the complete process of the proposed approach, an example (malignant) is shown in Fig.1. In Fig. 1(b), the image is fuzzified and divided into lattices; in Fig.1 (c), the ROI is determined by the proposed ROI generation method; by the proposed classification method, the case is successfully classified as a malignant tumor, since the classification ratio is $86.99 \%$.

To evaluate the overall performance of the proposed method, five descriptive statistics (accuracy (ACC), sensitivity (SEN), specificity (SPE), positive predictive value (PPV) and negative predictive value (NPV)) are used [2, 3]. Define the number of correctly and incorrectly classified malignant tumors as TP and FN, the number of correctly and incorrectly classified benign tumors as TN and FP, respectively, the five descriptive statistics can be calculated as: (1) ACC = $(\mathrm{TP}+\mathrm{TN}) /(\mathrm{TP}+\mathrm{TN}+\mathrm{FP}+\mathrm{FN})$; (2) $\mathrm{SEN}=$ $\mathrm{TP} /(\mathrm{TP}+\mathrm{FN})$; (3) SPE = TN/(TN+FP); (4) $\mathrm{PPV}=\mathrm{TP} /(\mathrm{TP}+\mathrm{FP}) ;$ (5) $\mathrm{NPV}=$
$\mathrm{TN} /(\mathrm{TN}+\mathrm{FN})$, and they are shown in Table 1 . The size of the window, $d_{w i n}$, is selected as 40. The overall accuracy is $93.75 \%$, and it means the classification is very accurate. The sensitivity is $95.0 \%$, it means that most of the malignant tumor can be detected by the proposed method. The specificity and the PPV (92.31\% and 93.44\%) show that the number of the benign tumors which are incorrectly classified as malignant is very low, i.e., with the proposed method, very few benign tumors need further examination.

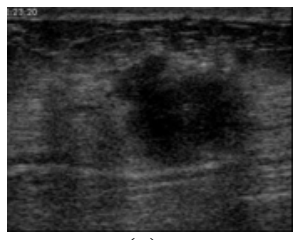

(a)

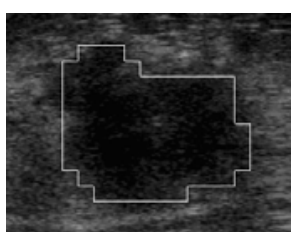

(c)

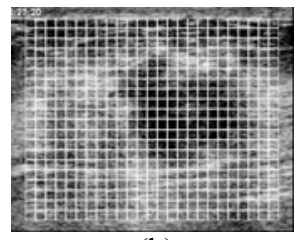

(b)

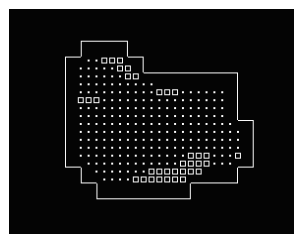

(d)
Fig. 1 (a) The original image; (b) The fuzzified image and the lattices; (c) The generated ROI; (d) The classification results of the classification checkpoints, the checkpoints correctly classified are marked by the solid white dots, and the checkpoints classified wrongly are marked by the white hollow rectangles.

Table 1 . The descriptive statistics

\begin{tabular}{|c|c|c|}
\hline Window Size & ACC & SEN \\
\hline$d_{\text {win }}=40$ & $93.75 \%$ & $95.0 \%$ \\
\hline SPE & PPV & NPV \\
\hline $92.31 \%$ & $93.44 \%$ & $94.12 \%$ \\
\hline
\end{tabular}




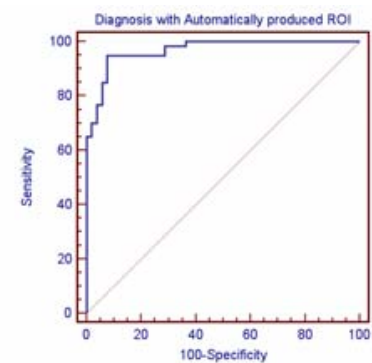

Fig. 2 The ROC curve of the classification ratio

The receiver operator characteristic (ROC) curve which evaluates the performance is shown in Fig. 2. The $A_{z}$ value is 0.968 and the $95 \%$ confidence interval is $[0.917,0.992]$. The indexes validate that the proposed method can classify breast tumors effectively.

\section{Conclusions}

In this paper, a novel classification approach of BUS images is proposed. The characteristics of the proposed method can be summarized:

(1) Unlike most of existing methods, the proposed method focused on finding a credible ROI instead of precisely segmenting the BUS image.

(2) The novel feature extraction and classification strategy focused on modeling the local texture features around the classification checkpoints in the ROIs and classifying the images based on the classification results of the checkpoints.

The experimental results demonstrate that the proposed approach is very effective and useful for classifying the breast tumors using BUS images.

\section{Acknowledgement}

This work is supported, in part, by National Science Foundation of China; grant numbers: 60573071; 60873142; 30670546

\section{References}

[1] K. Drukker, M. L. Giger, C. J. Vyborny, and E. B. Mendelson, "Computerized detection and classification of cancer on breast ultrasound," Acad Radiol, vol. 11, no. 5, pp. 52635, 2004.

[2] R. F. Chang, W. J. Wu, W. K. Moon, D. R. Chen, "Improvement in breast tumor discrimination by support vector machines and speckle-emphasis texture analysis," Ultrasound in Med. \& Bio., vol. 29, no. 5, pp. 679-686, 2003.

[3] D. R. Chen, R. F. Chang, C. J. Chen, M. F. Ho, S. J. Kuo, S. T. Chen, S. J. Hung, W. K. Moon, "Classification of breast ultrasound images using fractal feature," Clinical Imaging, vol. 29, pp. 235-245, 2005.

[4] N. Piliouras, I. Kalatzis, N. Dimitropoulos, D. Cavouras, "Development of the cubic least squares mapping linear-kernel support vector machine classifier for improving the characterization of breast lesions on ultrasound, " Comperterized Medical Imaging and Graphics, vol. 28, pp. 247-255, 2004.

[5] P. S. Rodrigues, G. A. Graldi, M. Provenzano, M. D. Faria, R. F. Chang and J. S. Suri, "A New Methodology Based on q-Entropy for Breast Lesion Classification in 3-D Ultrasound Images", Proceedings of the $28^{\text {th }}$ IEEE EMBS annual international conference, pp. 10481051, 2006.

[6] J. A. Noble, and D. Boukerroui, "Ultrasound image segmentation: a survey," IEEE Trans. Med. Imag., vol.25, no.8 pp. 987-1010, Aug. 2006.

[7] S. K. Pal and D. Majumder, "Fuzzy mathematical approach to pattern recognition”, John Wiley \& Sons, 1986. 
[8] H. D. Cheng, X. J. Shi, R. Min, L. M. Hu, X. P. Cai and H. N. Du, "Approaches for Automated Detection and Classification of Masses in Mammograms”, Pattern Recognition, Vol. 39, pp. 646-668, 2006.

[9] V. Vapnik, "Statistical learning theory,” Wiley-Interscience, 1998. 